in preparation. Only a few typical results are given here :

\begin{tabular}{|c|c|c|c|c|}
\hline Material. & $\begin{array}{l}t \text { from theory } \\
\text { in } \mathrm{cm} .\end{array}$ & $\begin{array}{l}t \text { from obs. } \\
\text { in } \mathrm{cm} \text {. }\end{array}$ & $\begin{array}{l}\text { Mean } \\
\text { diameter of } \\
\text { flbres from } \\
\text { theory in } \\
\text { cm. }\end{array}$ & $\begin{array}{l}\text { Fibre } \\
\text { diameter } \\
\text { directly } \\
\text { measured } \\
\text { in cm. }\end{array}$ \\
\hline Boxwood & $\begin{array}{l}0.0746 ; 0.0759 \\
0.058 ; 0.051\end{array}$ & $\begin{array}{l}0.075 \\
0.055\end{array}$ & $6.55 \times 10^{-4}$ & $6.58 \times 10^{-1}$ \\
\hline & & & \multirow{3}{*}{\multicolumn{2}{|c|}{$\begin{array}{l}\text { The thickness of grain } \\
\text { deduced =906.24 } \times 10^{-8} \\
\text { cm. and this is } 251 \times \\
3.610 \text { A., where } 3.610 \mathrm{~A} \\
\text { is the value from X-ray } \\
\text { measurement. } \\
\text { The thickness of grain } \\
\text { deduced }=80554 \times 10^{-8} \\
\text { cm.y and this is } 200 \times \\
3.027 \text { A., where } 3.02 \mathrm{~A} \text {. } \\
\text { is the value from X-ray } \\
\text { mesourement. }\end{array}$}} \\
\hline Copper & $\begin{array}{l}0.0333 ; 0.0338 \\
0.0449 ; 0.0665\end{array}$ & $\begin{array}{r}0.033 \\
0.045 ; 0.070\end{array}$ & & \\
\hline Slate & $\begin{array}{l}0.0442 ; 0.042 \\
0.0381 ; 0.0370\end{array}$ & $\begin{array}{l}0.045 ; 0.040 \\
0.038 ; 0.037\end{array}$ & & \\
\hline
\end{tabular}

The wood fibres were prepared by the Schulze process, and their mean diameters were measured directly under a microscope.

Physics Department, Science College,

$$
\text { K. Prosad. }
$$

Patna, India, Nov. 21.

1 Zeits. tech. Physik, 5, 9, pp. 369-378.

Phil. Mag., vol. 7, p. 548, March 1929.

\section{Systems of Four Immiscible Liquid Layers.}

Systams of three immiscible layers are sufficiently uncommon to be noteworthy, and no system of four layers appears to have been described (excluding systems containing free mercury). Nor is this remarkable when it is recalled, first, that practically all $d r y$ organic liquids are completely miscible, almost the only exceptions being polyhydric alcohols; secondly, that a system of four liquid phases requires the existence of four substances or solutions, which taken three at a time yield at a fixed temperature four systems each of three liquid phases, the immiscibility of which is in no case destroyed on saturation with the fourth component. The majority of the hitherto-described systems of three liquid phases (of which the system wateraniline-hexane is typical) owe their existence to the properties of a substance such as aniline or succinic nitrile, which is not completely miscible with water, but itself absorbs sufficient water to destroy its miscibility with another solvent, such as hexane or carbon disulphide, which absorbs only traces of water.

A study of systems containing soaps revealed a different type of three-layer system. When an electrolyte is added to an aqueous soap solution in equilibrium with an organic solvent not completely miscible with it (or when the soap concentration is increased beyond a certain limit), one of three things may happen :

(1) The soap is salted out as solid curd. layer.

(3) The salted-out soap absorbs water and solvent to form a third layer immiscible with either lye or solvent.

The system sodium oleate, sodium chloride, ethyl acetate, water, illustrates all three possibilities: the first, for example, at $25^{\circ}$, the second at $55^{\circ}$ for high salt concentrations, the third for lower salt concentrations at the same temperature. These three-layer systems differ from the type previously mentioned in that two of the layers are predominantly aqueous in composition. Since such systems can be prepared using either aniline or hexane as the solvent, and since wet aniline, hexane, and water are mutually immiscible, the conditions are fulfilled for a system of four liquid layers, provided the presence of soap in at least three of them does not destroy their immiscibility. Suitable pro- portions do, in fact, yigld a four-phase system at room temperatures ; this is most easily prepared by mixing the organic liquids and oleic acid (which are miscible in the absence of water), and adding sufficient aqueous sodium hydroxide to saponify the fatty acid and salt out the soap. The following proportions yield ap. proximately equal volumes of the four layers:

$$
\begin{aligned}
& \text { Hexane (or light petroloum) . - } 12 \text { c.c. } \\
& \text { Aniline . . . } 7 \text { c.e. } \\
& \text { Oleic Acid . } \quad . \quad \text {. } \quad \text {. } \quad \text {. } 0.5 \text { c.c. } \\
& \text { Alcohol : : } 1.5 \text { c.c. } \\
& \text { Aqueous Sodium Hydroxide }-0.8 \mathrm{~N} 10 \text { c.c. }
\end{aligned}
$$

The alcohol is not essential to the system, but reduces the time required for separation of the layers from hours to minutes. An increase of temperature or of electrolyte concentration renders miscible the hexane and aniline layers (first and third from the top), which then form the top layer.

This four-phase system contains five components (excluding the alcohol), which is one more than the minimum required by the phase rule. Although both aniline and hexane could probably be replaced by other liquids, there seems to be no possibility of producing a four-phase system of this type with only four components.

It need scarcely be added that the addition of mercury gives a five-layer system-a unique scientific curiosity.

Research Laboratory,

Chelsea Polytechnic, S.W.3,

$$
\text { Dec. } 9 .
$$

\section{The Longitudinal Distribution of Photoelectrons.}

Formule of the type

$$
P(\theta) d \theta \propto(A+B \cos \theta) \sin ^{3} \theta d \theta
$$

as a first approximation to more complex forms have been proposed as illustrating the existence of longitudinal asymmetry in the spatial distribution of photoelectrons. ${ }^{1}$ Here $P(\theta) d \theta$ is the probability of photoelectric emission between angles $\theta$ and $(\theta+d \theta)$ to the ray, $A$ is usually unity and $B$ some function of the frequency of the incident radiation and of the atomic number of the atoms upon which the radiation falls. The angle of bipartition $\theta_{b}$ is then obtained from the relation

$$
\int_{0}^{\theta_{b}}(A+B \cos \theta) \sin ^{3} \theta d \theta=\int_{\theta_{b}}^{\pi}(A+B \cos \theta) \sin ^{3} \theta d \theta
$$

and the average forward momentum

$$
\begin{gathered}
m v \overline{\cos \theta}=m v \int_{0}^{\pi}(A+B \cos \theta) \sin ^{3} \theta \cos \theta d \theta \\
/ \int_{0}^{\pi}(A+B \cos \theta) \sin ^{3} \theta d \theta
\end{gathered}
$$

I should like to point out that from another point of view the distribution formula (I) does not give the information concerning $\theta_{b}$ and $m v \overline{\cos \theta}$ that is ascribed to it. A similar expression can be built up from quite simple principles, which, however, preclude us from performing any integration outside the limits 0 to $\pi / 2$.

Assuming the wave mechanical principle of interference $P(\theta) \propto \sin ^{2} \theta$ and that independent groups of photoelectrons originate from a small plane circular area of radius $b$ normal to the ray and a small spherical volume of radius $a$ respectively, situated at the origin, we could write down at once the whole probability

$$
P(\theta) d \theta \propto\left(A^{\prime} \pi a^{2}+B^{\prime} \pi b^{2} \cos \theta\right) \sin ^{3} \theta d \theta
$$

where $A^{\prime}$ and $B^{\prime}$ are factors independent of $\theta$.

From the way in which the problem is stated and because of the inclusion of the cosine term involving 